\title{
Linking parental mediation practices to adolescents' problematic online screen use: A systematic literature review
}

\author{
PHILIP NIELSEN ${ }^{1,2}$, NICOLAS FAVEZ ${ }^{2}$, HOWARD LIDDLE ${ }^{3}$ and HENK RIGTER ${ }^{4}$ \\ ${ }^{1}$ Centre Mail, Fondation Phénix, Geneva, Switzerland \\ ${ }^{2}$ Unité de Psychologie Clinique des Relations Interpersonnelles, FPSE, Université de Genève, Geneva, Switzerland \\ ${ }^{3}$ Departments of Public Health Sciences and Psychology, Center for Treatment Research on Adolescent Drug Abuse, University of Miami \\ Miller School of Medicine, Miami, FL, USA \\ ${ }^{4}$ Department of Child and Adolescent Psychiatry, Leiden University Medical Centre (LUMC), Leiden, The Netherlands
}

(Received: April 28, 2019; revised manuscript received: August 10, 2019; second revised manuscript received: October 19, 2019; accepted: October 20, 2019)

\begin{abstract}
Background and aims: To remedy problematic Internet use (PIU) and problematic online gaming (POG) in adolescents, much is expected from efforts by parents to help youths to contain their screen use. Such parental mediation can include (a) refraining from acting, (b) co-viewing or co-gaming with the teen, (c) active mediation, and (d) restrictive mediation. We evaluated if parental mediation practices are linked to PIU and POG in adolescents. Methods: For a systematic literature review, we searched for publications presenting survey data and relating parental mediation practices to levels of PIU and/or POG in adolescents. The review's selection criteria were met by 18 PIU and 9 POG publications, reporting on 81.002 and 12.915 adolescents, respectively. We extracted data on gaming problems, mediation interventions, study design features, and sample characteristics. Results: No type of parental mediation was consistently associated with lower or elevated problematic screen use rates in the adolescents. Refraining from parental mediation tended to aggravate screen use problems, whereas active mediation (talking to the teen) may mitigate such problems in PIU, but less clearly in POG. The link of restrictive mediation with problematic screen use varied from positive to negative, possibly depending on type of restriction. In both PIU and POG, family cohesion was related to lower rates of the problem behavior concerned and family conflict to higher rates. Discussion and conclusions: Parental mediation practices may affect problematic screen use rates for better or worse. However, research of higher quality, including observations of parent-teen interactions, is needed to confirm the trends noted and advance the critical issue of the possible association between PIU, POG, and family interactions.
\end{abstract}

Keywords: adolescents, problematic Internet use, problematic online gaming, parental mediation, family cohesion, systematic literature review

\section{INTRODUCTION}

As television started to dominate mid-20th century family life, communication research focused on how parents could protect their children against the negative impact this medium might have (Clark, 2011). The term "parental mediation" was coined, i.e., behavioral strategies parents can apply to contain their child's television use (Valkenburg, Krcmar, Peeters, \& Marseille, 1999). With the rapid expansion of the Internet, parental concerns have shifted to their child's online screen use (Boyd \& Hargittai, 2013).

Today, a major challenge for parents is how they can protect their youths from engaging in harmful online activities (Livingstone \& Helsper, 2008). Internet use offers young people information and entertainment and allows them to learn skills, to have social contacts, and to express their feelings and opinions (Louge, 2006). Yet, online activities may become problematic for some adolescents. We increasingly see such teens in our clinical practice, where we also face their parents who need guidance. Looking for effective forms of guidance, we wondered what can be learned from the television-era parental mediation practices. To find out, we conducted a systematic literature review.

\section{Problematic screen use in adolescents}

There is no consensus how to define Internet use problems. The term "Internet addiction" has been proposed (Young, 2017), but so far this concept has not been accepted as a diagnosis in the fifth edition of the Diagnostic and Statistical Manual of Mental Disorders (DSM-5) and 11th edition of the International Classification of Diseases (ICD-11;

\footnotetext{
* Corresponding author: Philip Nielsen; Unité de Psychologie Clinique des Relations Interpersonnelles, FPSE, Université de Genève, 40, bd Pont-d'Arve, 1211, Geneva, Switzerland; Phone: +41 7950641 13; Fax: +41 2237993 59; E-mail: philip.nielsen@ unige.ch
}

This is an open-access article distributed under the terms of the Creative Commons Attribution-NonCommercial 4.0 International License, which permits unrestricted use, distribution, and reproduction in any medium for non-commercial purposes, provided the original author and source are credited, a link to the CC License is provided, and changes - if any - are indicated. 
WHO) catalogs of mental health disorders. Alternative labels have been suggested: Internet use being compulsive (Koning, Peeters, Finkenauer, \& van den Eijnden, 2018; van den Eijnden, Spijkerman, Vermulst, van Rooij, \& Engels, 2010), excessive (Kalmus, Blinka, \& Olafsson, 2015), or pathological (Chng, Li, Liau, \& Khoo, 2015). We prefer the term "problematic" (Bleakley, Ellithorpe, \& Romer, 2016; Gomez, Rial, Brana, Golpe, \& Varela, 2017; Kammerl \& Wartberg, 2018), with acronym PIU referring to problematic Internet use. For behavior to qualify as PIU, a case must meet personal and social harm criteria. Just accessing Internet frequently or even "excessively" does not suffice to consider a youth to be a problematic user (Aarseth et al., 2017). PIU, in our view, is characterized by impaired control over screen use, with screen use taking precedence over other life interests and daily activities, and by inability to stop or de-escalate screen use despite experiencing negative consequences.

Apart from PIU in general, the literature also reports on specific problematic screen-related behaviors, such as smartphone (Roberts, Yaya, \& Manolis, 2014) and social media use (Koning et al., 2018). A special category is online gaming.

\section{Problematic online gaming in adolescents}

The research community is divided if online gaming can develop into addiction (Aarseth et al., 2017). Nevertheless, DSM-5 has entered Internet Gaming Disorder (IGD) as a provisional diagnosis, to be validated in further research. ICD-11 lists Gaming Disorder (online or offline) as an established disorder. Yet, to be on the safe side, we use here the term "problematic online gaming" (POG). POG has the same characteristics as just defined for PIU, with "gaming" to be substituted for "screen use."

\section{Definition of parental mediation practices targeting screen use}

Parental mediation practices focusing on screen use have been modeled after those from the television era. Five types of mediation have been distinguished (Collier et al., 2016; Livingstone \& Helsper, 2008; Nathanson, 1999, 2001, 2002; Nikken \& Jansz, 2006):

1. No mediation. The parents take no action. They do not limit or encourage their teen's screen use.

2. Co-using, referring to a parent and the adolescent accessing an Internet-based screen jointly, without the parent resorting to criticism. We use the term co-viewing for shared Internet use such as watching a movie together via a streaming site, and co-gaming for playing games together (Nikken \& Jansz, 2006).

3. Active mediation, i.e., a parent talking with the adolescent about screen use and screen content without indulging in criticism.

4. Monitoring. For instance, the parents may check which sites the adolescent accesses and which games he or she plays and for how long, without discussing this knowledge with the teen - that would be active mediation. Just knowing that his or her parents are paying attention may influence an adolescent's screen use (Benrazavi, Teimouri, \& Griffiths, 2015).
5. Restrictive mediation. This consists of setting rules and limits to the adolescent's access to screens. The restrictions - mild or severe (even punitive) - can be verbal, but also technical, i.e., parents employing software to limit Internet access and time on the Internet (Benrazavi et al., 2015).

These categories are not mutually exclusive. Parents may combine practices.

\section{Aims of this study}

We here report on a systematic review assessing any links between the listed parental mediation practices on one hand and the rate of (a) PIU and (b) POG among adolescents on the other. We examined if diverse parental mediation practices might be beneficial or harmful in influencing problematic screen use.

The review was carried out in two parts: one addressing PIU and the other POG. We treated PIU and POG as separate entities, for two reasons. Unlike POG, PIU has not yet been accepted as a disorder by DSM-5 or ICD-11. Second, there is no conclusive evidence to consider POG as being part of PIU. The two conditions may be similar or distinct (Király, Nagygyörgy, Griffiths, \& Demetrovics, 2014).

Quite a few of the publications we sampled for this dual review also reported on another set of factors that may influence the development and persistence of PIU and POG in teens, i.e., family cohesion and family conflict (Bonnaire, Liddle, Har, Nielsen, \& Phan, 2019; Bonnaire \& Phan, 2017). As the setting for parental mediation to take shape is the family, we decided to include, when mentioned, the association between family cohesion/conflict and PIU and POG rates.

\section{METHODS}

Both parts of the review adhere to the guidelines of the PRISMA Group (Moher, Liberati, Tetzlaff, \& Altman, 2009). We wrote a review protocol, listed search terms, and developed and pilot tested a PIU and POG coding form (all available from the author PN) to note down key characteristics of the selected studies, i.e., type (crosssectional, prospective, with or without a comparison sample) and the size of the study samples (the number of adolescents surveyed, and if relevant the number of parents), the country where the study had been carried out, and the setting from which the samples were taken [school (including college and university) and general population]. For each sample of adolescents, we recorded the adolescents' mean age and age range, the gender distribution, and comorbidity findings. Household income and other indicators were used to estimate the socioeconomic status of the families at issue. Furthermore, we registered the method to assess PIU or POG. We took note of the type of mediation applied by the parents and how it was measured, and of any statistically significant correlations between these practices and the adolescent's screen use. Finally, we abstracted information on family cohesion and family conflict. 


\section{Inclusion and exclusion criteria}

We searched for publications issued between January 1 , 2000 and December 31, 2018 that presented original quantitative research data linking parental mediation practices to the adolescent's level of either Internet use, including phone and social media use, or online gaming. We sampled reports of cross-sectional and prospective studies with measures of (a) PIU or online gaming and (b) parental mediation practices. Publications were excluded if written in a language other than English, Dutch, German, and French. Excluded too were publications on site content (cyberbullying, sexual harassment, and porn) rather than on PIU or POG. Case reports, literature reviews, and meta-analyses were not considered.

We focused on youths between 12 and 19 years of age. If these adolescents were part of a sample with a wider age range (across all selected PIU and POG publications, age ranged from 8 to 20 years), we included the whole sample if the group of 12-19 olds could not be parceled out.

The literature surveyed addressed parental attempts to prevent or mitigate adolescents' problematic screen use. We excluded publications if they considered relational and emotional parent-teen interactions, or parenting styles, but failed to present data on actual parental mediation practices. The dividing line between parental mediation and parental style is thin; we opted for a strict sampling approach, only selecting papers that clearly specified, which mediation practices had been investigated.

\section{Searching for publications}

We looked for relevant publications on PIU and POG (papers, books/book chapters, and $\mathrm{PhD}$ theses) in five databases, viz., Web of Science, Embase, Cochrane Database of Systematic Reviews, Medline/PubMed, and PsycINFO. The searches took place on April 25 and 26 of 2018, except for PsycINFO (first week of May 2018), with further searches done from May through December 2018. In addition, we consulted our self-maintained Dropbox file of Internet use and online gaming publications.

The search of Web of Science yielded most records (291), followed in number - after removing modest overlap (160 overlap cases in total) - by Medline/PubMed (207), PsychINFO (153), Embase (123), and Cochrane (11). In the first assessment round, the authors PN and HR independently from each other abstracted the Abstracts and Methods sections of all these publications using the coding form's list of "relevance criteria" (cross-sectional/prospective study design, being a research paper, involving adolescent cases, focus on problematic screen use, mentioning concrete mediation practices). There were a few cases (in the Web of Science search: 22 papers; 7\%) where the two reviewers differed in degree of certainty about selecting a paper ("yes" vs. "question mark"), but this was resolved in discussions.

We wrote the corresponding author of each identified publication, asking him or her for a copy of any other relevant published or non-published paper from the same research group. We also invited the authors to comment on our summary of their sampled study; one correction was received.
The first assessment round yielded 117 publications (67 Web of Science, 9 Embase, 8 PsychINFO, 4 Medline/Pubmed, 0 Cochrane, 29 further publications from our Dropbox file, and from the reference lists of selected papers). Both reviewers reassessed this initial selection of publications using all coding from entries including the full list of inclusion and exclusion criteria for PIU and POG. In this second round, they in full agreement identified 27 publications meeting the inclusion criteria, i.e., 18 for PIU and 9 for POG (Tables 1 and 2). Thus, 90 publications were excluded: 44 because they did not focus on parental mediation but rather on parenting style or general parent-child relationship issues, 19 for failing to present a measure of PIU/online gaming (11) or of parental mediation (8), and 11 because they addressed site content rather than screen use. Sixteen publications were excluded for other reasons (see Figure 1 for the flow diagram).

\section{Search terms}

We used synonyms for "adolescent" (youth, teen, teenager, and young adult), "Internet use" [social media, cell phone, screen use; addicted, problematic, compulsive, excessive use(s)/misuse/usage/user(s)/using], "Internet gaming problems" (disorder; addiction; problematic, compulsive, or excessive gaming), "parental mediation" (parental restriction, regulation, control, monitoring, and style). As an example, the Web of Science PIU + POG query was:

TS $=$ (teenager or adolescen* or teen* or "young adults" or youth*) AND TS = ("internet abuse" or "internet addict*" or "compulsive internet use" or "compulsive internet usage" or "excessive internet use" or "excessive internet usage" or "internet addiction disorder" or "internet dependenc*" or "internet misuse*" or "internet gaming disorder" or "internet over-use" or "internet overuse" or "online addiction" or "pathologic* internet usage" or "problematic online usage" or "gaming addict*" or "compulsive computer use" or "computer addict*" or "pathological computer use" or "smart phone addiction" or "smart phone addict*") AND TS = ("parental mediation" or "parental restriction" or "parental regulation" or "parental control" or "parental supervision" or "parental involvement" or "parental attitude*" or "family functio*" or "family cohesion" or "family relationships" or "parenting").

The full string of search terms for each database is available from the author PN.

\section{Assessing the quality of the selected studies}

For lack of a published checklist to rate the quality of this type of study, we developed our own assessment sheets. The one for PIU is shown in Appendix, the one for POG (available from the author $\mathrm{PN}$ ) is similar, substituting "POG" for "PIU." The forms addressed sampling methods, study design features, measurement methods, and completeness of reporting on key variables.

We deemed methods (tools/questionnaires) for evaluating PIU valid and reliable if they had been included in 
Nielsen et al.

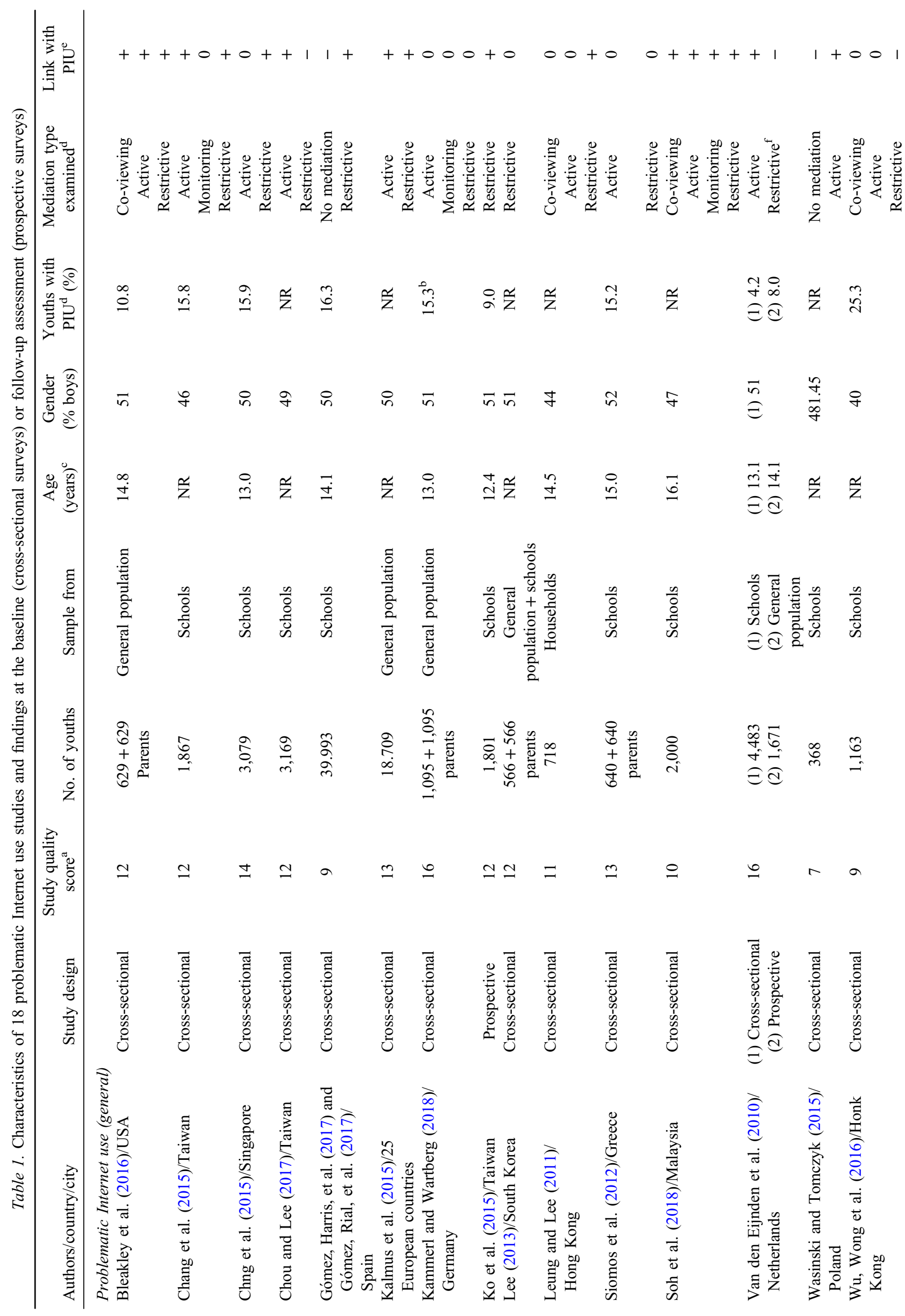




Table 1 of the review by Lortie and Guitton (2013). The
tools listed in that table scored well on validity and reliabil-
ity measures, except in some cases for criterion validity,
i.e., the capability to distinguish between normal and PIU.
We followed these reviewers' procedure for evaluating the
tools described in papers published from 2013 onward,
arriving at similar conclusions as reported in a second
review (Anderson, Steen, \& Stavropoulos, 2017). Tools
meeting the Lortie and Guitton lenient standard obtained

\section{RESULTS}

All selected publications, issued between 2010 and 2018, were journal papers. Table 1 lists the 18 PIU papers, including one on smartphone use and one on social media use. Table 2 presents the nine papers on POG.

The maximum quality score for a study was 20 (Appendix). The rating scales we developed are yet to be validated, so cut-off levels were not defined. Scores were similar in two separate ratings scheduled 3 months apart from each other. The quality scores for PIU studies varied from 7 to 16 (Table 1), those for POG studies from 9 to 12 (Table 2). Main reasons for low-quality scores were suboptimal survey design - cross-sectional rather than prospective in most cases - lack of (full) random sampling, failure to include a sample of parents, and inadequate measurement of PIU, POG, or of mediation practices.

\section{Research sites}

PIU. All studies were performed in either Europe (8) or East Asia (9), except for one US survey (Table 1). The European investigations were carried out in Germany, Greece, the Netherlands (2x), Poland, and Spain [two studies of one sample of adolescents (Gomez, Harris, Barreiro, Isorna, \& Rial, 2017; Gomez, Rial, et al., 2017)]. One survey covered 25 European countries. The Asian studies were from Hong Kong (2×), Malaysia (1×), Singapore (1×), South Korea $(2 x)$, and Taiwan $(3 x)$.

$P O G$. The core research groups were from Eastern Asia ( $3 \times$ South Korea, $2 \times$ China, $1 \times$ Malaysia, $1 \times$ Singapore, and $1 \times$ Taiwan), with two exceptions (1 France and 1 the Netherlands; see Table 2). 
Nielsen et al.

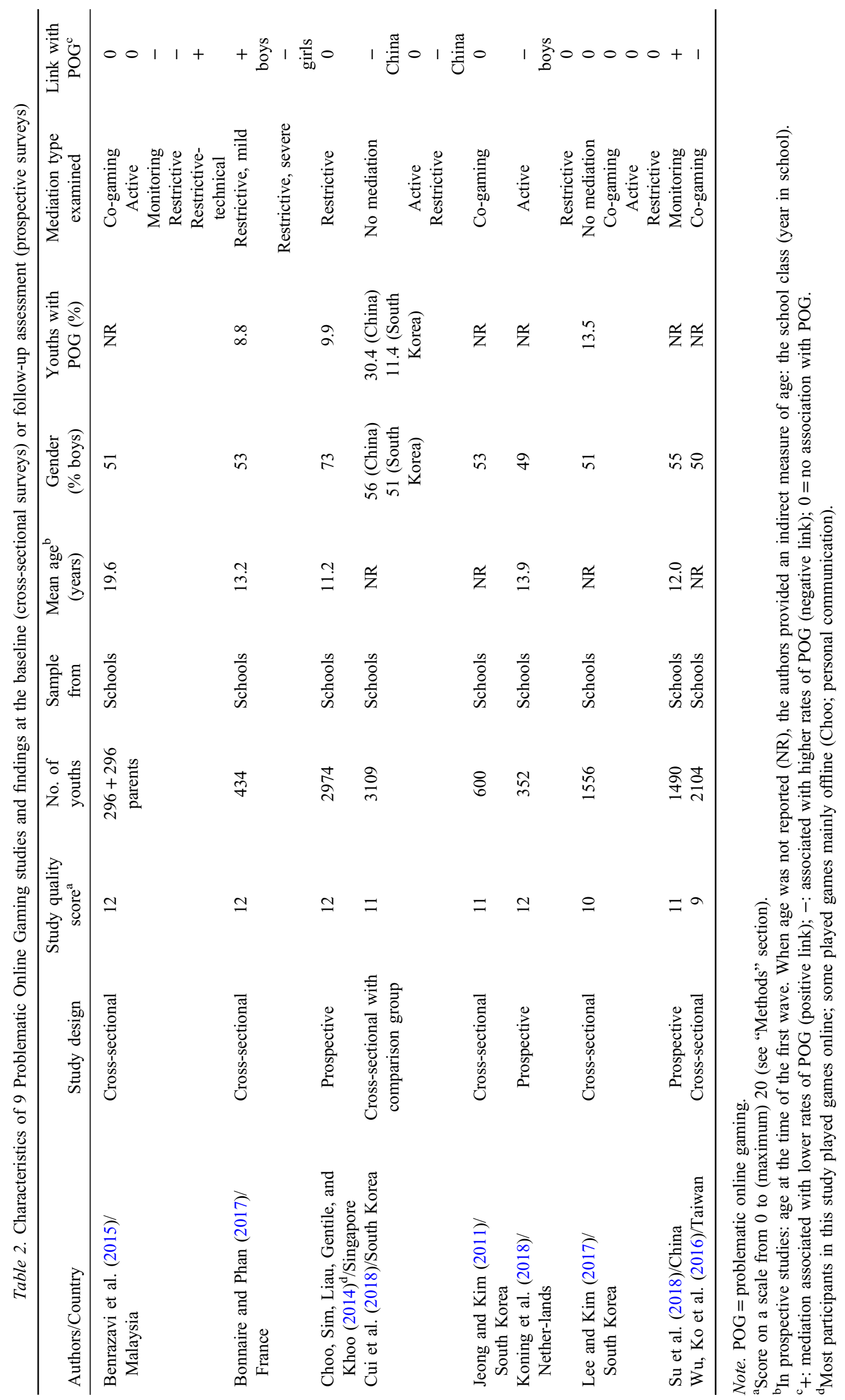




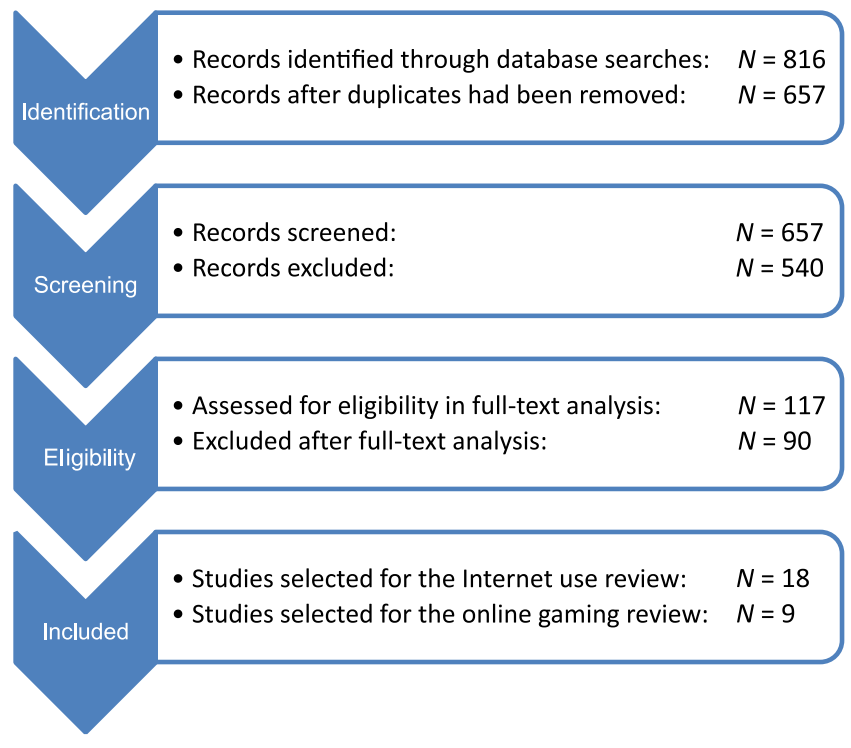

Figure 1. Study selection flow diagram

\section{Participants' characteristics and gaming-related factors}

PIU. A total of 81,002 adolescents were surveyed. They were mostly part of school convenience samples (13 out of 18 surveys). Five studies sampled adolescents from the general population.

The mean age of the adolescents ranged from 12.4 to 16.1 years. The proportion of boys varied from $40 \%$ to $52 \%$ and the proportion of adolescents meeting PIU criteria ranged from $4 \%$ to $25 \%$ (Table 1 ). Problematic screen use was more prevalent among boys than girls (e.g., Chang et al., 2015; Chng et al., 2015; Chou \& Lee, 2017, Wu, Ko, Wong, Wu, \& Oei, 2016). According to the single paper on problematic smartphone use (Lee, Kim, \& Choi, 2017), smartphone use was more problematic among girls than boys.

The socioeconomic status of the adolescent's family was measured in just two PIU studies (Chang et al., 2015; Wu, Wong, Yu et al., 2016) and two POG studies. In Wu, Wong, et al.'s (2016) survey, low family income was associated with higher teen PIU rates, but not in the other PIU survey or in the two POG studies reporting on social-economic status (Choo et al., 2014; Cui, Lee, \& Bax, 2018).

Adolescents' mental comorbidity was not assessed in any of the PIU and POG investigations, except for one PIU study that measured symptoms of depression (Chang et al., 2015). PIU youths had elevated rates of depression symptoms.

POG. These studies surveyed 12.915 adolescents, all from school convenience samples. Their mean age varied from 11.2 to 13.2 years, with one outlier of 19.6 years (Benrazavi et al., 2015) and the proportion of boys from $49 \%$ to $73 \%$. The prevalence of POG ranged from $9 \%$ to $30 \%$ (Table 2), with rates being higher for boys than girls (Bonnaire \& Phan, 2017; Choo et al., 2014; Cui et al., 2018; Lee \& Kim, 2017).

Three POG studies addressed the role of game genre (Bonnaire \& Phan, 2017; Cui et al., 2018; Lee \& Kim, 2017). Playing massively multiplayer online role-playing games, rather than other games, was associated with higher adolescent POG rates in France (Bonnaire \& Phan, 2017) and South Korea (Cui et al., 2018), but not in China where playing action games was most clearly associated with POG (Cui et al., 2018).

Frequent playing of online games correlated with a high rate of POG. Furthermore, playing games after midnight was associated with higher POG rates (Bonnaire \& Phan, 2017; Cui et al., 2018; Lee \& Kim, 2017), perhaps even more so when games were played on a mobile device (Lee \& Kim, 2017). Such "after-midnight" concerns were not reported in the PIU papers.

\section{Study design}

All studies were cross-sectional, except for three PIU and two POG surveys, which were (also) prospective (Tables 1 and 2). The follow-up assessments in the prospective studies were scheduled 6-12 months after the baseline assessment. Only one group of researchers enrolled a comparison group of adolescents (Cui et al., 2018). Five PIU studies and one POG study included a matching sample of parents (Tables 1 and 2).

Measures used to assess screen use, parental mediation, and family functioning

Problematic Internet use (PIU). All PIU and POG studies applied adolescent self-report measures of problematic screen use, but the questionnaires used to this end varied. All but one PIU studies applied an assessment tool with established value for measuring PIU. Six PIU surveys (Bleakley et al., 2016; Kammerl \& Wartberg, 2018; Lee, 2013; Leung \& Lee, 2011; Siomos et al., 2012; Wu, Ko, et al., 2016) administered (a version of) the Internet Addiction Test (IAT; Young, 1999). The IAT has been favorably reviewed by Lortie and Guitton (2013) as well as Moon, Hwang, Kim, Shin, Bae, and Kim (2018). Other diagnostic tools used, also appraised by Lortie and Guitton (2013) as being acceptable, were the Chen Internet Addiction Scale (CIAS; Chang et al., 2015; Chou \& Lee, 2017; Ko et al., 2015) and the Compulsive Internet Addiction Scale (CIUS; van den Eijnden et al., 2010).

Problematic gaming. The two European research groups (Bonnaire \& Phan, 2017; Koning et al., 2018) chose the well-tested Gaming Addiction Scale (GAS; Lemmens, Valkenburg, \& Peter, 2009). The Malaysian research group opted for the Problematic Online Gaming Questionnaire (Benrazavi et al., 2015), which has generally adequate psychometric properties (King et al., 2013). Cui et al. (2018) as well as Lee and Kim (2017) administered the South Korean Problematic Gaming Use Scale, which is adapted from the GAS. Some groups of researchers attempted to turn PIU tools (IAT and CIAS) into a POG tool by rewording PIU questions in POG terms or by adding POG questions (Jeong \& Kim, 2011; Su et al., 2018).

Most POG questionnaire items were phrased like DSM-5/ICD-11 symptoms of IGD, although not always covering the full set of DSM/ICD symptoms (Bonnaire \& Phan, 2017; Cui et al., 2018; Jeong \& Kim, 2011; Lee \& Kim, 2017; Su et al., 2018). 
Parental mediation. The sampled PIU and POG papers provided little information on the reliability and validity of the diverse tools employed for measuring parental mediation. Most of the questionnaires for assessing mediation were self-designed or locally designed, using the adolescents as sole source of information. One exception to this lack of well-established parental mediation measurement methods was the questionnaire developed in the EU Kids Online project, used by Chang et al. (2015; PIU), Chng et al. (2015; PIU), Kalmus et al. (2015; PIU), and by Benrazavi et al. (2015; POG). A second exception was the Parental Mediation Inventory developed by Lim and Cho (2011), as adopted by Cui et al. (2018; POG) as well as Lee and Kim (2017; POG).

Family cohesion and conflict. Ten out of 18 PIU and 6 out of 9 POG studies examined one or more aspects of the relationship between parents and their teen children. Diverse questions and questionnaires were used to assess the relationship between parents and child, with topics ranging from parent-teen communication, closeness, bonding, attachment, affection, joint social activities, but also conflict. We use here as summary terms: family cohesion (good relationships between parents and teens) and the opposite, family conflict. Most family functioning tools were self-made, limited to a few questions, and based on self-report by the adolescent, with incomplete evidence presented about the validity and reliability of these questionnaires. There were four exceptions: in PIU, the Adaptability, Partnership, Growth, Affection, and Resolve Scale (Ko et al., 2015; Wu, Wong, et al., 2016) and the EU Kids Online Questionnaire (Chng et al., 2015), and in $P O G$, the Family Relationship Index chosen by Bonnaire and Phan (2017).
The relationship between parental mediation and problematic screen use: Cross-sectional studies and first-wave assessments of prospective studies

Across studies, the number of mediation practices examined varied from one to five (Tables 1 and 2). Most studies were cross-sectional, yielding correlations between parental mediation and problematic screen use. These correlations between the two sets of variables suggest, but far from prove, that parental mediation may influence problematic screen use in adolescents. Figure 2 shows how often a parental mediation practice, across studies, was linked to lower (positive) or higher (negative) problematic screen use levels.

No mediation. This parental practice was examined in just two PIU and two POG studies (Tables 1 and 2). "No mediation" was associated with higher PIU (Gomez, Harris, et al., 2017; Gomez, Rial, et al., 2017; Wasinski \& Tomczyk, 2015) and POG rates [Cui et al., 2018 (Chinese, but not Korean youths)], except for one survey (Lee \& Kim, 2017).

Co-viewing and co-gaming. The role of co-viewing and co-gaming was analyzed in four PIU and four POG studies, with mixed results. In two PIU (Leung \& Lee, 2011; Wu, Wong et al., 2016) and three POG surveys (Benrazavi et al., 2015; Jeong \& Kim, 2011; Lee \& Kim, 2017), co-viewing/ co-gaming did not bear on problematic screen use rates. However, in three other studies, this mediation practice correlated with lower adolescent problematic screen use rates (Bleakley et al., 2016; Soh, Chew, Koay, \& Ang, 2018; Wu, Ko, et al., 2016).

Active mediation. PIU: Active mediation was associated with a lower prevalence of problematic screen use in six

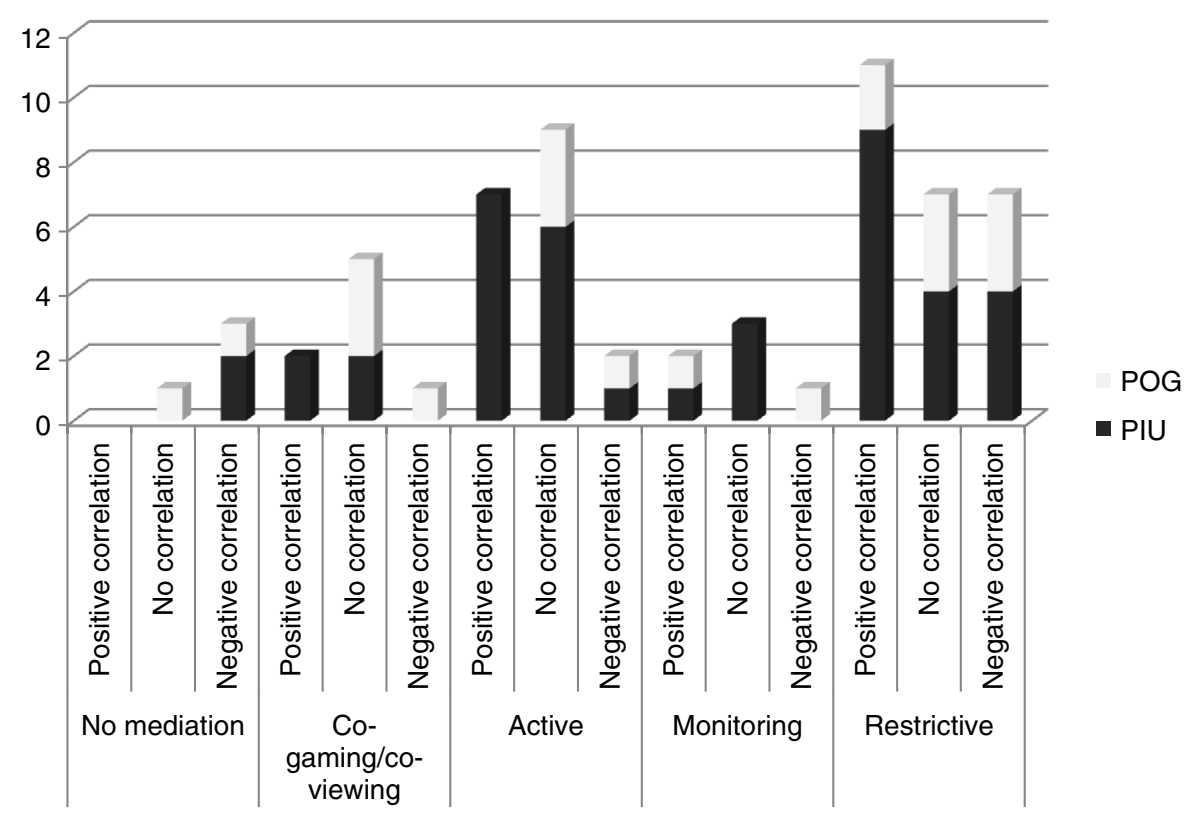

Figure 2. Nature of the correlations between distinct types of parental mediation practices and problematic screen use among adolescents. Five parental mediation practices as examined in problematic Internet use (PIU) studies and problematic online gaming studies (POG). Number of studies per correlation outcome. Positive correlation: the mediation practice was associated with lower rates of problematic screen use (PIU or POG). Negative correlation: the reverse, associated with higher rates. No correlation: no statistically significant association between mediation practice and problematic screen use. Not included in the PIU and PIU + POG graphs: the papers on problematic social media use (Koning et al., 2018) and problematic smartphone use (Lee et al., 2017) 
studies (Bleakley et al., 2016; Chang et al., 2015; Kalmus et al., 2015; Soh et al., 2018; van den Eijnden et al., 2010; Wasinski \& Tomczyk, 2015), with no association noted in four other studies (Kammerl \& Wartberg, 2018; Leung \& Lee, 2011; Siomos et al., 2012; Wu, Wong, et al., 2016). In the single study on problematic social media use (Koning et al., 2018), active mediation was associated with higher problem use levels in boys but not girls. No such link was observed in the paper on problematic smartphone use (Lee et al., 2017).

$P O G$ : In contrast, the POG studies yielded little evidence of a protective role of active mediation. In the four studies measuring this parental practice, three failed to find any link between active mediation and adolescents' rates of problematic gaming (Benrazavi et al., 2015; Cui et al., 2018; Lee \& Kim, 2017). The fourth study confirmed this lack of relation between active mediation and POG for girls, but among boys active mediation was associated with higher POG rates (Koning et al., 2018).

Monitoring. There was no consistent evidence in favor of monitoring. In three studies, this parental mediation practice was not related to PIU (Chang et al., 2015; Kammerl \& Wartberg, 2018) or to problematic smartphone use (Lee et al., 2017). Monitoring has been examined in just one cross-sectional POG survey; the practice was linked to higher POG rates (Benrazavi et al., 2015).

Restrictive mediation. The parental practice most often assessed in cross-sectional studies is restrictive mediation (Tables 1 and 2).

PIU: The results were mixed. In 6 out of 12 investigations, restrictive mediation was associated with lower PIU rates (Bleakley et al., 2016; Chang et al., 2015; Chng et al., 2015; Gomez, Harris, et al., 2017; Gomez, Rial, et al., 2017; Kalmus et al., 2015; Leung \& Lee, 2011), and in three instances with higher rates (Chou \& Lee, 2017; van den Eijnden et al., 2010; Wu, Wong, et al., 2016). Three surveys failed to find any correlation between restrictive mediation and PIU (Kammerl \& Wartberg, 2018; Lee, 2013; Siomos et al., 2012).

POG: The POG surveys provided little proof of a beneficial influence of restrictive mediation. Signs of a positive influence were noted for subsamples (boys: Bonnaire \& Phan, 2017; Chinese rather than Korean youths: Cui et al., 2018) or when parents applied a specific type of restriction (limiting screen access through technical means; Benrazavi et al., 2015). Apart from this, restrictive mediation was not linked to problematic gaming (Choo et al., 2014; Koning et al., 2018; Lee \& Kim, 2017) or rather correlated with higher POG rates, again in subsamples: Malaysian youths (non-technical restriction); girls subjected to severe, punitive restriction in France (Bonnaire \& Phan, 2017), and Chinese youths in a China - Korea comparison (Cui et al., 2018).

The relationship between parental mediation and problematic screen use: Prospective studies

Three of the PIU surveys were prospective, in part or in full (Ko et al., 2015; Koning et al., 2018; van den Eijnden et al., 2010), as were three of the POG surveys (Choo et al., 2014; Koning et al., 2018; Su et al., 2018). The prospective investigations included two or three assessment rounds, spaced 6 to 12 months apart.

PIU: In the Ko et al.'s (2015) study, 9.1\% of the adolescents who were ruled to be "non-addicts" at the baseline assessment developed PIU in the subsequent year, providing an estimate of the incidence of PIU. Factors associated with elevated rates of incident PIU were family conflict (see below) and lack of restrictive parental mediation. Van den Eijnden et al. (2010) carried out two parallel studies: one cross-sectional and one prospective. In both approaches, active mediation was associated with lower PIU rates and restrictive mediation with higher PIU rates. Koning et al. (2018) reported the opposite: active mediation predicting later problematic screen use, restrictive mediation predicting screen use to be less troubled than at baseline. However, the latter investigation targeted problematic social media use rather than PIU in general.

POG: If an adolescent had developed POG, according to the first assessment, the parents increased their mediation efforts in the ensuing 6-12 months (Koning et al., 2018; Su et al., 2018), but this was not reflected in decreased POG rates at the follow-up assessments (Choo et al., 2014; Koning et al., 2018).

\section{Family cohesion}

PIU: In the studies that measured the influence of family variables, low family cohesion and high family conflict including interparental conflict - correlated with elevated rates of PIU (Chang et al., 2015; Chng et al., 2015; Soh et al., 2018; Wu, Wong, et al., 2016). The negative influence of family conflict was confirmed in a prospective investigation (Ko et al., 2015).

$P O G$ : Family cohesion may protect an adolescent against developing POG, as suggested by both cross-sectional (Bonnaire \& Phan, 2017; Cui et al., 2018; Lee \& Kim, 2017) and prospective investigations (Choo et al., 2014; Su et al., 2018). There were gender effects. In Choo et al.'s (2014) prospective survey, the bond between father and adolescent was more protective than the teen's bond with the mother. In another prospective investigation, a good relationship between father and adolescent again predicted lower rates of POG at the first follow-up assessment $(\mathrm{Su}$ et al., 2018). Family conflict, on the other hand, correlated with increased POG rates (Bonnaire \& Phan, 2017).

In the three prospective POG investigations, the acrossassessments relationship between family cohesion and POG rates was reciprocal (Choo et al., 2014; Koning et al., 2018; Su et al., 2018). In all three studies, parental mediation was associated with both family cohesion and POG rates, suggesting interactions between these three sets of factors.

\section{DISCUSSION AND CONCLUSIONS}

\section{Parental mediation}

We distinguished five types of parental mediation. The first was "no mediation," a rarely examined approach. The few available data suggest that "no mediation" may be harmful, i.e., it is associated with elevated rates of PIU and POG. 
Perhaps, refraining from parental mediation is a sign of indifferent parenthood or a disengaged parent-teen relationship (Lee \& Kim, 2017; Wasinski \& Tomczyk, 2015).

A second approach is co-viewing and co-gaming. Coviewing was an effective mediation practice in the television era (Livingstone \& Helsper, 2008). We could not confirm this for online screen use. Co-viewing/co-gaming had no clear impact on PIU or POG rates.

Third, active mediation: parents talking with their child about screen use without intervening by setting rules. This practice worked well for television-watching (Clark, 2011; Livingstone \& Helsper, 2008). Similarly, in most of the PIU studies, active mediation correlated with lower rates of problematic screen use, so might be beneficial. The same cannot be said about POG; we failed to find any link between active mediation and problematic gaming. This raises the question if PIU and POG are alike or not. POG may be a disorder on its own or a category of PIU (Király et al., 2014). Our findings do not confirm that PIU and POG are related but are not strong enough to prove they are distinct.

A fourth practice, monitoring, may protect young people against abusing drugs (Dishion \& McMahon, 1998), excessive screen use (Gentile, Reimer, Nathanson, Walsh, \& Eisenmann, 2014), and online harassment (Khurana, Bleakley, Jordan, \& Romer, 2015). In our review, though, monitoring was not consistently associated with any pattern of problematic screen use by adolescents. This failure to establish a link may be due to inadequate research methodology - studies exclusively relying on self-report data - or on the vagueness of the concept "monitoring" as applied to online screen use (see below).

Restrictive mediation, the fifth approach, helps to prevent children from excessively watching television (Nathanson, 1999, 2002). In the sampled PIU and POG studies, restrictive mediation was the frequently examined parental mediation practice. The results were mixed, for both PIU and POG, alternating between beneficial, harmful, and ineffective. At present, there is no clear support for a role for restrictive mediation in aiding adolescents to contain their screen use.

\section{Family cohesion and conflict}

Family cohesion and its counterpart family conflict were repeatedly associated in adolescents with rates of both PIU and POG rates. We found family cohesion to be linked to lower rates of PIU and POG and family conflict to higher rates (cross-sectional studies confirmed in prospective studies). This was true even if parents imposed screen use restrictions. Perhaps, the adolescent does not oppose restrictive mediation, mild or severe, when he or she feels comfortable with his or her parents (Choo et al., 2014).

Thus, family cohesion and family conflict appear to be importantly protective and risk factors, respectively, for the development and persistence of both PIU and POG. Perhaps, these factors moderate or mediate the effects of parental mediation, or vice versa, but this is speculative as only a few studies have addressed this issue to some extent (Choo et al., 2014; Koning et al., 2018; Su et al., 2018).

\section{Other factors}

We could not establish any impact of age, as the adolescents, except for one POG study, were all young teens, with mean ages mostly ranging from 12 to 15 years. POG youths tended to be somewhat younger than PIU youths. Consistent with other research, our review shows that boys are more likely to develop PIU and POG than girls (Anderson et al., 2017; Király et al., 2014). Gender may influence an adolescent's choice which online activities to pursue. For instance, Lee and Kim (2017) report that boys are interested in action-packed games, which more easily give rise to POG than other games, whereas girls prefer casual games. Perhaps, this gender-related game genre preference may explain the difference in POG liability between boys and girls. Game genre, an important variable (Anderson et al., 2017; Stavropoulos et al., 2018), was unfortunately neglected in most POG surveys.

The PIU studies were not very informative as to which Internet use practices may contribute to the rise and persistence of problematic screen use, apart from accessing screens frequently and for long periods of time (e.g., Bleakley et al., 2016; Kalmus et al., 2015). POG studies provided more insight. At night, when the parents go to bed, the influence of parental interventions may wear off. After-midnight gaming is associated with the development of POG in adolescents (e.g., Bonnaire \& Phan, 2017).

In our review, countries/continents did not differ much in PIU rates among adolescents, which were mostly in the range of $10 \%-20 \%$. They also did not differ much for POG (range: $8 \%-30 \%$ ). The latter was against our expectations; in Saunders et al.'s (2017) review, prevalence rates of adolescent POG were said to vary from under $1 \%$ to $10 \%$ in Western countries to $10 \%$ to $15 \%$ or higher in East Asian countries. This discrepancy in findings may be due, at least in part, to methodological differences, such as sampling procedures, between surveys addressing different study questions.

\section{Possible reasons for failing to find strong evidence in favor of parental mediation}

We found limited evidence that some parental mediation practices may help mitigating PIU and/or POG. Overall, however, there was no strong case for any mediation practice, except perhaps for active mediation in PIU. This may mean that parental mediation is weakly effective at best, but before reaching this conclusion, there are confounding factors to consider.

First, the methodological quality of the sampled surveys was into the low to high middle range of the assessment form for POG, and slightly higher for PIU, but never excellent, meaning that the reported outcomes cannot be fully trusted. We inferred which mediation practices had been implemented from the questionnaires filled out by the adolescent and occasionally by the parents. Just relying on self-reports is risky (Fan et al., 2006). Actual observations of parent-child interactions are needed, which were missing in all studies. Random sampling and prospective assessments should be the norm in future studies. 
One other reason for failing to find strong evidence for the importance of parental mediation may have been the vagueness of the concept of parental mediation in online screen use context. One example is monitoring, not or ill-defined in most papers concerned. One paper offered practical suggestions for operationalizing monitoring as a mediation practice. The researchers asked parents to describe their monitoring efforts, if any: check the teen's profile on a gaming platform; ask the teen's friends about the gaming site, requirements, and networks; check out the game website; and read messages sent and received by the teen (Benrazavi et al., 2015). A second example of a vaguely defined parental mediation practice is restriction. One type of restriction was parents telling the adolescent which home rules applied for accessing the Internet and playing games: when, where, and for how long. Other types included locking away the equipment, installing technical aids to limit screen access, or punishing the adolescent for breaking rules. With this variation of interventions, "restrictive mediation" is a confusing concept. Research tools are needed to define restrictive mediation more precisely; a rare example of this approach is given by Benrazavi et al. (2015).

Ideas about proper mediation practices may vary between cultures. This may also have contributed to the heterogeneity of our findings. In the perception of Chinese researchers, Western parents (or Malaysian parents; Benrazavi et al., 2015) tend to respectfully talk about their children's personal choices and they may provide advice, whereas Chinese parents rather emphasize parental authority and restrictive mediation, and more readily supervise their children's behavior (Su et al., 2018). Hypotheses like these should be tested in sound observational investigations.

Mediation practices from the television era may be outdated in the Internet era (Benrazavi et al., 2015), partly because present-day adolescents are much more skilled and knowledgeable in using screens and bypassing access blockades. With rapidly evolving technology, one would expect more fine-tuned tools to emerge to prevent and control POG. New parental mediation practices may emerge, such as "participatory learning," which involves parents and children interacting together with and through digital media (Clark, 2011).

\section{Limitations of this study}

Although our literature searches were extensive, we may have missed relevant publications. We were unable to read five of the identified papers, which were written in Chinese or Korean. The authors of these papers also published in English, so their work is represented in this review. A second limitation may have been a lack of fine-tuning in the search terms used to trace publications on specific forms of Internet use. We identified just one parental mediation paper on teen problematic smartphone use (Lee et al., 2017) and one on social media use (Koning et al., 2018). We doubt, though, if incomplete search terms were the cause of this modest harvest. More likely, parental mediation has been neglected so far in research on problematic phone and social media use (Kuss \& Griffiths, 2011; Roberts et al., 2014).

\section{Strengths of this study}

This review fully conformed to the guidelines of the PRISMA Group (Moher et al., 2009). We were very strict in sampling papers on parental mediation, excluding papers referring to mediation without offering a solid method to measure relevant interventions. Furthermore, we excluded papers on just parenting style and parental attitudes and on ill-defined interventions. The papers passing our finegrained selection sieve really were relevant for addressing our review questions.

\section{Conclusions}

Of the five parental mediation practices, 'no mediation' tended to be harmful in PIU and POG cases and active mediation tended to be beneficial in PIU cases. A close relationship between parents and adolescents protected teens against problematic screen use; family conflict was linked to the reverse, i.e., elevated rates of PIU and POG. Future studies of parental mediation practices should consider the relational-emotional parent-teen context (family cohesion).

Most of the evidence collected was from cross-sectional studies, which cannot establish causal relationships. The results of the few prospective studies were generally in line with those of the cross-sectional investigations. More prospective studies are needed, with random sampling and with assessments covering three or more follow-up years, as has been done by the research groups of Chng and Shek (Chng et al., 2015; Shek, Zhu, \& Ma, 2018). Reliable and valid questionnaires should be designed to measure parental mediation and family cohesion. Most importantly, the emphasis in studies should switch from convenience sample epidemiology to (also) observations of real interactions between parents and their teens.

Overall, thus far, the literature cannot deliver a strong case in favor of parental mediation. This seemingly contrasts with the body of evidence from the television era. However, the parental mediation practices used in the television era may not work out the same way in the Internet era. The impact of interventions may be weaker, family structure has changed, most family members have access to private screens, and perhaps most critically, the nature of the Internet use and gaming phenomena is fundamentally different from television viewing. Parental mediation practices that worked well in the television era may work differently or are inadequate in the Internet era. This is not to say that there is no role for parental mediation in present times, although perhaps the term "mediation," so tied to the television era, should be substituted for another term, such as parental influence. Technological developments have been so fast as to bedazzle parents and teens. Parental practices need to be adapted and fine-tuned to present-day circumstances.

Funding sources: No financial support was received for this study.

Authors' contribution: All authors were instrumental in developing the study concept. The review was designed and carried out by PN and HR and supervised by NF. 
HL helped in selecting publications to be reviewed. PN and HR wrote the paper, with critical input and assistance with data interpretation from HL and NF. All authors had full access to all study data and take responsibility for the integrity of the data and the accuracy of the data analysis.

Conflict of interest: The authors declare no conflict of interest.

Acknowledgements: The authors would like to thank Mafalda Burri, Service de référence, University of Geneva Library, for assisting in identifying search terms and for carrying out the database searches.

\section{REFERENCES}

The publications marked with ** are part of the PIU review and those marked with * are of the POG review.

Aarseth, E., Bean, A. M., Boonen, H., Colder Carras, M., Coulson, M., Das, D., Deleuze, J., Dunkels, E., Edman, J., Ferguson, C. J., Haagsma, M. C., Helmersson Bergmark, K., Hussain, Z., Jansz, J., Kardefelt-Winther, D., Kutner, L., Markey, P., Nielsen, R. K. L., Prause, N., Przybylski, A., Quandt, T., Schimmenti, A., Starcevic, V., Stutman, G., Van Looy, J., \& Van Rooij, A. J. (2017). Scholars' open debate paper on the World Health Organization ICD-11 Gaming Disorder proposal. Journal of Behavioral Addictions, 6(3), 267-270. doi:10.1556/2006.5.2016.088

Anderson, E. L., Steen, E., \& Stavropoulos, V. (2017). Internet use and problematic Internet use: A systematic review of longitudinal research trends in adolescence and emergent adulthood. International Journal of Adolescence and Youth, 22(4), 430-454. doi:10.1080/02673843.2016.1227716

*Benrazavi, R., Teimouri, M., \& Griffiths, M. D. (2015). Utility of parental mediation model on youth's problematic online gaming. International Journal of Mental Health and Addiction, 13(6), 712-727. doi:10.1007/s11469-015-9561-2

**Bleakley, A., Ellithorpe, M., \& Romer, D. (2016). The role of parents in problematic Internet use among US adolescents. Media and Communication, 4(3), 24-34. doi:10.17645/mac. v4i3.523

Bonnaire, C., Liddle, H. A., Har, A., Nielsen, P., \& Phan, O. (2019). Why and how to include parents in the treatment of adolescents presenting Internet gaming disorder. Journal of Behavioral Addictions, 8(2), 201-212. doi:10.1556/2006.8. 2019.27

*Bonnaire, C., \& Phan, O. (2017). Relationships between parental attitudes, family functioning and Internet gaming disorder in adolescents attending school. Psychiatry Research, 255(Suppl. C), 104-110. doi:10.1016/j.psychres.2017.05.030

Boyd, D., \& Hargittai, E. (2013). Connected and concerned: Variation in parents' online safety concerns. Policy \& Internet, 5(3), 245-269. doi:10.1002/1944-2866.POI332

**Chang, F. C., Chiu, C. H., Miao, N. F., Chen, P. H., Lee, C. M., Chiang, J. T., \& Pan, Y. C. (2015). The relationship between parental mediation and Internet addiction among adolescents, and the association with cyberbullying and depression. Comprehensive Psychiatry, 57, 21-28. doi:10.1016/j.comppsych. 2014.11.013
**Chng, G. S., Li, D. D., Liau, A. K., \& Khoo, A. (2015). Moderating effects of the family environment for parental mediation and pathological Internet use in youths. Cyberpsychology Behavior, and Social Networking, 18(1), 30-36. doi:10.1089/cyber.2014.0368

*Choo, H., Sim, T., Liau, A. K. F., Gentile, D. A., \& Khoo, A. (2014). Parental influences on pathological symptoms of video-gaming among children and adolescents: A prospective study. Journal of Child and Family Studies, 24(5), 1429-1441. doi:10.1007/s10826-014-9949-9

**Chou, C., \& Lee, Y. H. (2017). The moderating effects of Internet parenting styles on the relationship between Internet parenting behavior, Internet expectancy, and Internet addiction tendency. Asia-Pacific Education Researcher, 26(3-4), 137-146. doi:10.1007/s40299-017-0334-5

Clark, L. S. (2011). Parental mediation theory for the digital age. Communication Theory, 21(4), 323-343. doi:10.1111/j.14682885.2011.01391.x

Collier, K. M., Coyne, S. M., Rasmussen, E. E., Hawkins, A. J., Padilla-Walker, L. M., Erickson, S. E., \& Memmott-Elison, M. K. (2016). Does parental mediation of media influence child outcomes? A meta-analysis on media time, aggression, substance use, and sexual behavior. Developmental Psychology, 52(5), 798-812. doi:10.1037/dev0000108

*Cui, J., Lee, C., \& Bax, T. (2018). A comparison of 'psychosocially problematic gaming' among middle and high school students in China and South Korea. Computers in Human Behavior, 85, 86-94. doi:10.1016/j.chb.2018.03.040

Dishion, T. J., \& McMahon, R. J. (1998). Parental monitoring and the prevention of child and adolescent problem behavior: A conceptual and empirical formulation. Clinical Child and Family Psychology Review, 1(1), 61-75. doi:10.1023/A:10218 00432380

Fan, X. T., Miller, B. C., Park, K. E., Winward, B. W., Christensen, M., Grotevant, H. D., \& Tai, R. H. (2006). An exploratory study about inaccuracy and invalidity in adolescent selfreport surveys. Field Methods, 18(3), 223-244. doi:10.1177/ $152822 X 06289161$

Gentile, D. A., Reimer, R. A., Nathanson, A. I., Walsh, D. A., \& Eisenmann, J. C. (2014). Protective effects of parental monitoring of children's media use: A prospective study. JAMA Pediatrics, 168(5), 479-484. doi:10.1001/jamapediatrics. 2014.146

**Gomez, P., Harris, S. K., Barreiro, C., Isorna, M., \& Rial, A. (2017). Profiles of Internet use and parental involvement, and rates of online risks and problematic Internet use among Spanish adolescents. Computers in Human Behavior, 75, 826-833. doi:10.1016/j.chb.2017.06.027

**Gomez, P., Rial, A., Brana, T., Golpe, S., \& Varela, J. (2017). Screening of problematic Internet use among Spanish adolescents: Prevalence and related variables. Cyberpsychology, Behavior, and Social Networking, 20, 259-267. doi:10.1089/ cyber.2016.0262

*Jeong, E. J., \& Kim, D. H. (2011). Social activities, self-efficacy, game attitudes, and game addiction. Cyberpsychology, Behavior, and Social Networking, 14(4), 213-221. doi:10.1089/ cyber.2009.0289

**Kalmus, V., Blinka, L., \& Olafsson, K. (2015). Does it matter what mama says: Evaluating the role of parental mediation in European adolescents' excessive Internet use. Children \& Society, 29(2), 122-133. doi:10.1111/chso.12020 
**Kammerl, R., \& Wartberg, L. (2018). Zusammenhänge zwischen problematischer Internetnutzung im Jugendalter und Medienerziehung in der Familie [Interrelations between adolescent problematic Internet use and parental Internet mediation]. Praxis der Kinderpsychologie und Kinderpsychiatrie, 67(2), 134-153. doi:10.13109/prkk.2018.67.2.134

Khurana, A., Bleakley, A., Jordan, A. B., \& Romer, D. (2015). The protective effects of parental monitoring and Internet restriction on adolescents' risk of online harassment. Journal of Youth and Adolescence, 44(5), 1039-1047. doi:10.1007/s10964-014-0242-4

King, D. L., Haagsma, M. C., Delfabbro, P. H., Gradisar, M., \& Griffiths, M. D. (2013). Toward a consensus definition of pathological video-gaming: A systematic review of psychometric assessment tools. Clinical Psychology Review, 33(3), 331-342. doi:10.1016/j.cpr.2013.01.002

Király, O., Nagygyörgy, K., Griffiths, M. D., \& Demetrovics, Z. (2014). Problematic online gaming. In K. P. Rosenberg \& L. C. Feder (Eds.), Behavioral addictions. Criteria, evidence, and treatment (pp. 61-97). Amsterdam, The Netherlands: Elsevier/ Academic Press.

**Ko, C. H., Wang, P. W., Liu, T. L., Yen, C. F., Chen, C. S., \& Yen, J. Y. (2015). Bidirectional associations between family factors and Internet addiction among adolescents in a prospective investigation. Psychiatry and Clinical Neurosciences, 69(4), 192-200. doi:10.1111/pen.12204

***Koning, I. M., Peeters, M., Finkenauer, C., \& van den Eijnden, R. J. J. M. (2018). Bidirectional effects of Internet-specific parenting practices and compulsive social media and Internet game use. Journal of Behavioral Addictions, 7(3), 624-632. doi:10.1556/2006.7.2018.68

Kuss, D. J., \& Griffiths, M. D. (2011). Online social networking and addiction - A review of the psychological literature. International Journal of Environmental Research and Public Health, 8, 3528-3552. doi:10.3390/ijerph8093528

*Lee, C., \& Kim, O. (2017). Predictors of online game addiction among Korean adolescents. Addiction Research \& Theory, 25(1), 58-66. doi:10.1080/16066359.2016.1198474

**Lee, H., Kim, J. W., \& Choi, T. Y. (2017). Risk factors for smartphone addiction in Korean adolescents: Smartphone use patterns. Journal of Korean Medical Science, 32(10), 1674-1679. doi:10.3346/jkms.2017.32.10.1674

**Lee, S.-J. (2013). Parental restrictive mediation of children's Internet use: Effective for what and for whom? New Media \& Society, 15(4), 466-481. doi:10.1177/1461444812452412

Lemmens, J. S., Valkenburg, P. M., \& Peter, J. (2009). Development and validation of a Game Addiction Scale for Adolescents. Media Psychology, 12(1), 77-95. doi:10.1080/ 15213260802669458

**Leung, L., \& Lee, S. N. (2011). The influences of information literacy, Internet addiction and parenting styles on Internet risks. New Media \& Society, 14(1), 117-136. doi:10.1177/ 1461444811410406

Lim, S., \& Cho, Y. (2011). Parents' and peers' mediation effects on adolescents' game play. Journal of Cybercommunication Academic Society, 28, 173-218.

Livingstone, S., \& Helsper, E. J. (2008). Parental mediation of children's Internet use. Journal of Broadcasting \& Electronic Media, 52(4), 581-599. doi:10.1080/08838150802437396

Lortie, C. L., \& Guitton, M. J. (2013). Internet addiction assessment tools: Dimensional structure and methodological status. Addiction, 108(7), 1207-1216. doi:10.1111/add.12202
Louge, N. (2006). Adolescents and the Internet. Research facts and findings. ACT for Youth Center of Excellence, Cornell University. Retrieved from http://www.actforyouth.net/resources/ rf/rf_internet_1006.pdf

Moher, D., Liberati, A., Tetzlaff, J., \& Altman, D. (2009). Preferred reporting items for systematic reviews and metaanalyses: The PRISMA statement. Annals of Internal Medicine, 151(4), 264-269. doi:10.7326/0003-4819-151-4200908180-00135

Moon, S. J., Hwang, J. S., Kim, J. Y., Shin, A. L., Bae, S. M., \& Kim, J. W. (2018). Psychometric properties of the Internet Addiction Test: A systematic review and meta-analysis. Cyberpsychology, Behavior, and Social Networking, 21(8), 473-484. doi:10.1089/cyber.2018.0154

Nathanson, A. I. (1999). Identifying and explaining the relationship between parental mediation and children's aggression. Communication Research, 26(2), 124-143. doi:10.1177/ 009365099026002002

Nathanson, A. I. (2001). Parent and child perspectives on the presence and meaning of parental television mediation. Journal of Broadcasting \& Electronic Media, 45(2), 201-220. doi:10.1207/s15506878jobem4502_1

Nathanson, A. I. (2002). The unintended effects of parental mediation of television on adolescents. Media Psychology, 4(3), 207-230. doi:10.1207/S1532785XMEP0403_01

Nikken, P., \& Jansz, J. (2006). Parental mediation of children's videogame playing: A comparison of the reports by parents and children. Learning, Media and Technology, 31(2), 181-202. doi:10.1080/17439880600756803

Roberts, J. A., Yaya, L. H. P., \& Manolis, C. (2014). The invisible addiction: Cell-phone activities and addiction among male and female college students. Journal of Behavioral Addictions, 3(4), 254-265. doi:10.1556/JBA.3.2014.015

Saunders, J. B., Hao, W., Long, J., King, D. L., Mann, K., FauthBuhler, M., Rumpf, H. J., Bowden-Jones, H., RahimiMovaghar, A., Chung, T., Chan, E., Bahar, N., Achab, S., Lee, H. K., Potenza, M., Petry, N., Spritzer, D., Ambekar, A., Derevensky, J., Griffiths, M. D., Pontes, H. M., Kuss, D., Higuchi, S., Mihara, S., Assangangkornchai, S., Sharma, M., Kashef, A. E., Ip, P., Farrell, M., Scafato, E., Carragher, N., \& Poznyak, V. (2017). Gaming disorder: Its delineation as an important condition for diagnosis, management, and prevention. Journal of Behavioral Addictions, 6(3), 271-279. doi:10.1556/2006.6.2017.039

Shek, D. T. L., Zhu, X., \& Ma, C. M. S. (2018). The influence of parental control and parent-child relational qualities on adolescent Internet addiction: A 3-year longitudinal study in Hong Kong. Frontiers in Psychology, 9, 642. doi:10.3389/fpsyg. 2018.00642

**Siomos, K., Floros, G., Fisoun, V., Evaggelia, D., Farkonas, N., Sergentani, E., Lamprou, M., \& Geroukalis, D. (2012). Evolution of Internet addiction in Greek adolescent students over a two-year period: The impact of parental bonding. European Child \& Adolescent Psychiatry, 21(4), 211-219. doi:10.1007/ s00787-012-0254-0

**Soh, P. C.-H., Chew, K. W., Koay, K. Y., \& Ang, P. H. (2018). Parents vs peers' influence on teenagers' Internet addiction and risky online activities. Telematics and Informatics, 35(1), 225-236. doi:10.1016/j.tele.2017.11.003

Stavropoulos, V., Anderson, E. E., Beard, C., Latifi, M. Q., Kuss, D., \& Griffiths, M. (2018). A preliminary cross-cultural study of 
Hikikomori and Internet gaming disorder: The moderating effects of game-playing time and living with parents. Addictive Behaviors Reports, 9, 100137. doi:10.1016/j.abrep.2018.10.001

*Su, B., Yu, C., Zhang, W., Su, Q., Zhu, J., \& Jiang, Y. (2018). Father-child longitudinal relationship: Parental monitoring and Internet gaming disorder in Chinese adolescents. Frontiers in Psychology, 9, 95. doi:10.3389/fpsyg.2018.00095

Valkenburg, P. M., Krcmar, M., Peeters, A. L., \& Marseille, N. M. (1999). Developing a scale to assess three styles of television mediation: "instructive mediation," "restrictive mediation," and "social coviewing". Journal of Broadcasting \& Electronic Media, 43(1), 52-66. doi:10.1080/08838159909364474

**van den Eijnden, R. J., Spijkerman, R., Vermulst, A. A., van Rooij, T. J., \& Engels, R. C. (2010). Compulsive Internet use among adolescents: Bidirectional parent-child relationships. Journal of Abnormal Child Psychology, 38(1), 77-89. doi:10.1007/s10802-009-9347-8

**Wasinski, A., \& Tomczyk, L. (2015). Factors reducing the risk of Internet addiction in young people in their home environ- ment. Children and Youth Services Review, 57, 68-74. doi:10.1016/j.childyouth.2015.07.022

**Wu, C. S. T., Wong, H. T., Yu, K. F., Fok, K. W., Yeung, S. M., Lam, C. H., \& Liu, K. M. (2016). Parenting approaches, family functionality, and Internet addiction among Hong Kong adolescents. BMC Pediatrics, 16(1), 130. doi:10.1186/s12887016-0666-y

*Wu, J. Y. W., Ko, H. C., Wong, T. Y., Wu, L. A., \& Oei, T. P. (2016). Positive outcome expectancy mediates the relationship between peer influence and Internet gaming addiction among adolescents in Taiwan. Cyberpsychology, Behavior, and Social Networking, 19(1), 49-55. doi:10.1089/cyber. 2015.0345

Young, K. S. (1999). Internet addiction: Symptoms, evaluation and treatment. Innovations in Clinical Practice: A Source Book, 17(17), 351-352.

Young, K. S. (2017). Internet addiction in children and adolescents: Risk factors, assessment, and treatment. New York, NY: Springer Publishing Company. 


\section{APPENDIX}

The quality of survey reports on the link between parental mediation and adolescent problematic Internet use: A rating scale

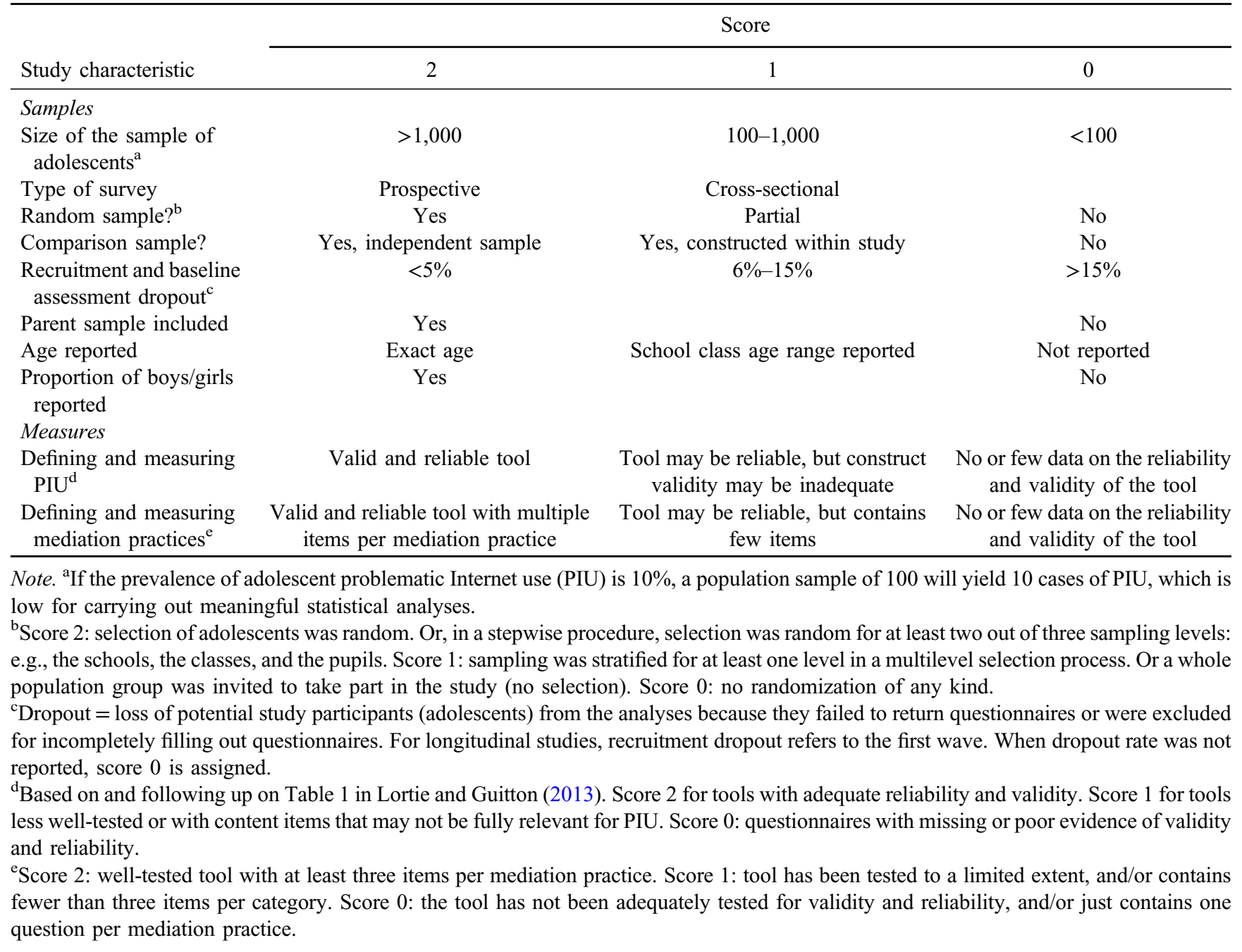

